ÖWAV-TAGUNGEN, ÖWAV-SEMINARE

Workshopreihe „Mit EMAS zu ökologisch aktiven Gemeinden, Verbänden und öffentlichen Einrichtungen", Workshop 1

Termin: 18.-19. Jänner 2010 (Workshop 2: 23.-24. März, Workshop 3: 8.-9. Juni 2010) Ort: wird noch bekanntgegeben Mitveranstaltung: Lebensministerium, Österreichischer Gemeindebund Leitung: Mag. Hermine Dimitroff-Regatschnig, DI Dr. Christian Plas

Kosten (+ $20 \%$ USt.): ÖWAV-Mitglieder/ Mitglieder des Österreichischen Gemeindebunds Euro 500,--, Nichtmitglieder Euro 700,- (2 Personen/Teilnehmer, für die gesamte Workshopreihe!)

Risikomanagement und Vorsorge für kritische Wasserinfrastruktur

Termin: 27. Jänner 2010

Ort: Universität Innsbruck

Leitung: Univ.-Prof. DI Dr. Wolfgang

Rauch

Kosten (+ $20 \%$ USt.): ÖWAV-Mitglieder

Euro 200,-, Nichtmitglieder Euro 350,-

\section{AEVG-ÖWAV-Fachtagung 2010}

„Wiederverwendung - Schlagwort oder neuer Maßstab?"“

Termin: 28. Jänner 2010

Ort: Flughafen Graz-Thalerhof

Kosten (+ $20 \%$ USt.): ÖWAV-Mitglieder Euro 120,-, Nichtmitglieder Euro 220,-

Abwässer aus Gewerbe und Industrie - Indirekt- und Direkteinleiter

Termin: 24.-25. Februar 2010 Ort: Technische Universität Wien Mitveranstalter: TU Wien, Institut für Wassergüte, Ressourcenmanagement und Abfallwirtschaft

Leitung: o.Univ.-Prof. DI Dr. Dr. h.c. Helmut Kroiß

\section{Verpackungsverordnung neu}

Termin: 4. März 2010

Ort: Bundesamtsgebäude, Wien Mitveranstalter: Lebensministerium, VÖEB und Industriellenvereinigung Leitung: Dr. Christian Keri

Kosten (+ $20 \%$ USt.): ÖWAV-Mitglieder Euro 200,-, Nichtmitglieder Euro 350,-
Vergaberecht für die Praxis

Termin: 11. März 2010

Ort: Bundesamtsgebäude, Wien Leitung: RA MMag. Dr. Claus Casati

Workshopreihe „Mit EMAS zu ökologisch aktiven Gemeinden, Verbänden und öffentlichen Einrichtungen", Workshop 2

Termin: 23.-24. März 2010 (Workshop 3: 8.-9. Juni 2010)

Ort: wird noch bekanntgegeben Mitveranstaltung: Lebensministerium, Österreichischer Gemeindebund Leitung: Mag. Hermine Dimitroff-Regatschnig, DI Dr. Christian Plas

Kosten (+ $20 \%$ USt.): ÖWAV-Mitglieder/ Mitglieder des Österreichischen Gemeindebunds Euro 500,-, Nichtmitglieder Euro 700,- (2 Personen/Teilnehmer, für die gesamte Workshopreihe!)

Österreichische Abfallwirtschaftstagung 2010 „Abfallwirtschaft im Wandel - Werte, Märkte und Strukturen"

Termin: 21.-23. April 2010

Ort: Wirtschaftskammer Eisenstadt

Mitveranstalter: Lebensministerium Kosten (+ $20 \%$ USt.): ÖWAV-Mitglieder Euro 350,-, Nichtmitglieder Euro 490,-

Fettabscheideranlagen - Vorstellung ÖWAV-Regelblatt 39 - Bemessung, Kennzeichnung, Betrieb

Termin: 20. Mai 2010

Ort: Kärnten (Ort wird noch bekanntgegeben)

Workshopreihe „Mit EMAS zu ökologisch aktiven Gemeinden, Verbänden und öffentlichen Einrichtungen", Workshop 3

Termin: 8.-9. Juni 2010

Ort: wird noch bekanntgegeben

Mitveranstaltung: Lebensministerium,

Österreichischer Gemeindebund

Leitung: Mag. Hermine Dimitroff-Regatschnig, DI Dr. Christian Plas

Kosten (+ $20 \%$ USt.): ÖWAV-Mitglieder/ Mitglieder des Österreichischen Gemeindebunds Euro 500,-, Nichtmitglieder Euro 700,- (2 Personen/Teilnehmer, für die gesamte Workshopreihe!)
Kanalmanagement 2010

Termin: 10. Juni 2010

Ort: BOKU, Wien

Leitung: Univ.-Prof. DI Dr. Raimund Haberl, Univ.-Ass. DI Dr. Thomas Ertl

Weitere Informationen und Anmeldung: Gesellschaft für Wasser- und Abfallwirtschaft GmbH des ÖWAV, 1010 Wien, Marc-Aurel-Straße 5, Martin Waschak, Tel. 01/535 57 20-75, waschak@oewav.at oder www.oewav.at $>>$ Veranstaltungen

Aufgrund der Voranmeldung erhalten Sie das detaillierte Veranstaltungsprogramm.

\section{ÖWAV-KURSE}

\section{ABWASSER}

\section{Klärwärter-Grundkurs}

Termin: 8.-26. Februar 2010

Ort: Großrußbach

Kosten (+ $20 \%$ USt.): ÖWAV-Mitglieder Euro 1.480,-, Nichtmitglieder Euro 1.720,-

\section{Mikroskopie-Grundkurs}

Termin: 15.-17. Februar 2010 Ort: Wien

Kosten (+ $20 \%$ USt.): ÖWAV-Mitglieder Euro 570,-, Nichtmitglieder Euro 720,-

\section{Mikroskopie-Spezialkurs}

Termin: 18.-19. Februar 2010

Ort: Wien

Kosten (+ $20 \%$ USt.): ÖWAV-Mitglieder Euro 400,-, Nichtmitglieder Euro 550,-

\section{Klärwärter-Grundkurs}

Termin: 1.-19. März 2010

Ort: Großrußbach

Kosten (+ $20 \%$ USt.): ÖWAV-Mitglieder Euro 1.480,-, Nichtmitglieder Euro 1.720,-

\section{8. ÖWAV-Kanalreinigungskurs}

Termin: 8.-10. März 2010

Ort: Innsbruck

96. Maschinentechnischer Kurs für Klärwärter

Termin: 8.-12. März 2010

Ort: Linz-Asten

\section{7. ÖWAV-Klärwärterkurs (50-500 EW)}

Termin: 15.-26. März 2010

Ort: Rappoltschlag (NÖ) 
Kosten (+ $20 \%$ USt.): ÖWAV-Mitglieder Euro 1.190,--, Nichtmitglieder Euro 1.230,-

\section{Laborpraktikum für Klärwärter}

Termin: 22.-26. März 2010

Ort: Linz-Asten

Kosten (+ $20 \%$ USt.): ÖWAV-Mitglieder Euro 910,-, Nichtmitglieder Euro 1.060,-

\section{Klärwärter-Grundkurs}

Termin: 12.-30. April 2010

Ort: Großrußbach

Kosten (+ $20 \%$ USt.): ÖWAV-Mitglieder

Euro 1.480,--, Nichtmitglieder Euro 1.720,-

20. VÖEB-ÖWAV-Kanaldichtheitsprüfungskurs

Termin: 11.-13. Oktober 2010

Ort: Anif

Informationen und Anmeldung: VÖEB, voeb@voeb.at, www.voeb.at

\section{ABFALL}

1. ÖWAV-Kurs „Elektronische Aufzeichnungen und Meldung von Abfallbilanzen"

Termin: 27. Jänner 2010

Ort: Wien

Kosten (+ $20 \%$ USt.): ÖWAV-Mitglieder Euro 310,-, Nichtmitglieder Euro 460,-

\section{2. ÖWAV-Kurs „Elektronische} Aufzeichnungen und Meldung von Abfallbilanzen"

Termin: 10. Februar 2010

Ort: Wien

Kosten (+ $20 \%$ USt.): ÖWAV-Mitglieder Euro 310,-, Nichtmitglieder Euro 460,-
3. ÖWAV-Ausbildungskurs ,Projektentwicklung, Betriebswirtschaft und Planungsgrundlagen von Biogasanlagen", Teil I-II

Termin: Teil I: 22.-24. Februar, Teil II: 15.-17. März 2010

Ort: Gleisdorf (Stmk.)

Kosten (+ $20 \%$ USt.): ÖWAV-Mitglieder Euro 1.140,-, Nichtmitglieder Euro 1.250,-

10. ÖWAV-Ausbildungskurs zum Abfallrechtlichen Geschäftsführer gemäß § 26 AWG 2002, Teil I-III

Termin: Teil I: 25.-27. Februar, Teil II: 4.-6. März, Teil III: 17.-19. März 2010 Ort: Wels

5. Ausbildungskurs „Mechanische Aufbereitung und thermische Behandlung von Abfällen"

Termin: 1.-5. März 2010

Ort: Nussdorf am Attersee

Kosten (+ $20 \%$ USt.): ÖWAV-Mitglieder Euro 990,-, Nichtmitglieder Euro 1.190,-

\section{BESCHNEIUNGSANLAGEN}

\section{3. ÖWAV-Schneimeister-Praxiskurs}

Termin: 19.-20. Jänner 2010

Ort: St. Anton am Arlberg und Lech am Arlberg

Kosten (+ $20 \%$ USt.): ÖWAV-Mitglieder/ Mitglieder des Marketingforums Seilbahnen Euro 750,--, Nichtmitglieder Euro 890,-

\section{HOCHWASSERSCHUTZANLAGEN}

ÖWAV-Kurs „Wartung und Betrieb von Hochwasserschutzanlagen

Termin: 3.-4. März 2010

Ort: Tulln
Weitere Informationen und Anmeldung: Gesellschaft für Wasser- und Abfallwirtschaft $\mathrm{GmbH}$ des ÖWAV, 1010 Wien, Marc-Aurel-Straße 5, Isabella Seebacher. Tel. 01/535 57 20-82, seebacher@ oewav.at oder www.oewav.at >> Veranstaltungen Aufgrund der Voranmeldung erhalten Sie das detaillierte Veranstaltungsprogramm.

\section{WEITERE VERANSTALTUN-} GEN: INLAND

1st IWA Austrian Young Water

Professionals Conference 2010

Termin: 9.-11. Juni 2010

Ort: Alte Schieberkammer, Wien

Auskunft: www.ywp-austria.at

\section{Depo-Tech 2010}

Termin: 3. bis 5. November 2010

Ort: Leoben

Auskunft: info@depotech.at, www. depotech.at

\section{WEITERE VERANSTALTUN- GEN: AUSLAND}

Internationales Symposium Auen und Hochwasser

Termin: 10.-11. Februar 2010

Ort: Ingolstadt

Auskunft: www.auen-und-hochwasser.de

Tagung des „Hydrologischen

Atlasses der Schweiz"

Termin: 18. März 2010

Ort: Casinotheater Winterthur

Auskunft: www.hades.unibe.ch 